\title{
Reiseimpressionen zwischen Differenz und Transkulturalität in Wolfgang Büschers Ein Frühling in Jerusalem
}

\author{
Inez Müller \\ Universität Paderborn
}

Zusammenfassung: Der Ich-Erzähler erschließt sich Jerusalem als transarealen Bewegungsraum, der für ethnische, religiöse und kulturelle Trennung steht, gleichzeitig aber auch als Ort der interkulturellen und interreligiösen Begegnung erlebt wird. Jerusalem kommt darüber hinaus in Einzelfällen auch als Kontaktzone hybrider Transformation literarisch zur Geltung. Der Beitrag untersucht Ein Frühling in Jerusalem als Reiseprosa des ethnologischen Blicks, in der divergierende Erzählversionen der Geschichte des 20./21. Jhds. im Kontext des Holocaust, der Diasporabewegungen und des Armenischen Völkermordes zur Sprache kommen.

Schlüsselbegriffe: Mythos, Interkulturalität, Diaspora, Flaneur, Labyrinth, Globalisierung

\begin{abstract}
The First-person-narrator explores and experiences Jerusalem as a transitory space in the meaning of ethnic, religious and cultural division and social segregation but at the same time as an intercultural and interreligious contact zone. The aspect of transculturality is of importance referring to the Mamilla Mall in Westjerusalem and the former huruma festivities. This article is focussing on the contemporary travelprose Ein Frühling in Jerusalem basing on the method of the ethnological look by telling the history of the Middle East in the historical context of the Holocaust, the Diaspora and the Armenian Genozide in a new way.
\end{abstract}

Keywords: myth, interculturality, diaspora, flaneur, labyrinth, globalisation 


\section{Einleitung}

Im Prosaband Ein Frühling in Jerusalem ${ }^{1}$ geht der autobiographisch schreibende Erzähler bei einem zweimonatigen Aufenthalt dem Mythos der Metropole Jerusalem nach. Dort sucht er das Gespräch mit allen ethnischen, sozialen und religiösen Gruppen. ${ }^{2}$ Auf diese Weise entsteht sowohl eine postmoderne Reise- als auch eine Großstadterzählung33, die Urbanität bewegungsgeschichtlich ${ }^{4}$ erfasst. Der Erzähler und Autor Büscher erkundet den kulturell geteilten Stadtraum Jerusalem in seinen innerstädtischen Grenzen und konfessionell getrennten Stadtvierteln. Der topographisch spezifische Ort bildet literarisch die Kontinuitäten aber auch die Brüche in der Stadtentwicklung Jerusalems bis in die Gegenwart ab. Dabei kommen divergierende Erinnerungsversionen der Genozide des 20. Jhds., der Exodusbewegungen und der Geschichte kolonialer wie auch neokolonialistischer Landnahmen zur Sprache: „[S]o setzt sich die Metropole vorrangig aus dem Neben-, Mitund Gegeneinander widersprüchlicher kultureller Stimmen, Geschichten und Traditionen zusammen".5 Die in Ein Frühling in Jerusalem aufgezeigten kulturellen Praktiken und Diskurse heterogener Art ${ }^{6}$ bewegen sich in einem Bereich zwischen Differenz, Interkulturalität und Transkulturalität77. In die Reiserzählung integrierte Immigrations-, Exil- und Auswanderungsgeschichten erschließen in Jerusalem eine kulturelle Topographie in steter Veränderung. Jerusalem stellt sich dem Erzähler wie auch dem Leser als heterogener „Fremd- und Konfliktraum“8 in Ambivalenz dar, denn Entwicklungen transkultureller Transformationen ${ }^{9}$ hinsichtlich Ver- und Durchmischung von Kulturen erweisen sich in Jerusalem wie andernorts in Israel als scheinbar unvereinbar mit regierungspolitischen Aktionen neointerventionistischer Gewaltausübung, die im Prosaband kritisch mit kommentiert ist. Nach Natan Sznaider ${ }^{10}$ wird der Diasporagedanke in Israel ideologisch und politisch eingesetzt, was Prozesse der Selbstabschottung11 zur Folge hat und die militante Siedlerbewegung (vgl. FJ 174ff) einflussreich macht. ${ }^{12}$ Der vielsprachige und multikulturelle Stadtraum ${ }^{13}$ Jerusalem steht in Büschers Reisetext für positiv konnotierte Transkulturalität, die Nationalitätsdiskurse überwinden helfen könnte, sofern diese gesellschaftliche Wirkung entfaltet. Historisch betrachtet funktioniert Jerusalem bereits transkulturell als Zielort unterschiedlicher Migrationsbewegungen. ${ }^{14}$ In 
welchem Maße Jerusalem dieses Potential an Transkulturalität in der Gegenwart zu nutzen versteht, erkundet Büscher in seinem Prosaband Ein Frühling in Jerusalem mit den literarischen und journalistischen Methoden des ethnologischen Blicks, des Interviews und der Geschichtslektüre (vgl. FJ 137). Damit leistet diese Erzählsammlung einen wesentlichen Beitrag zur kulturellen Topographie des Reisens im Nahen Osten.

\section{Der Reisende als Flaneur und Autor}

Der Erzähler bezeichnet sich selbst als Spaziergänger und Wanderer zwischen der Altstadt und den mehr oder weniger voneinander abgegrenzten religiösen Vierteln der Stadt Jerusalem, „[d]en blauen Koffer in der Hand, betrat ich durchs Jaffator die Heilige Stadt" (FJ 13). Als Steine Lesender (vgl. FJ 76) und Studierender schreibt sich der IchErzähler in die Tradition des Flaneurs der Moderne ein: „Ich streifte durch viele Konvente, lateinische, armenische und byzantinische samt kleiner Kirche, durch arabische und jüdische Wohnhöfe - sie glichen einander" (FJ 76). Wie die Denkfigur des Literaten und Flaneurs in Walter Benjamins Passagen-Werk ${ }^{15}$ erkundet auch der Erzähler in Ein Frühling in Jerusalem gehend, sehend und das Eigene erinnernd Jerusalem als die Fremde, indem er diese als Text zu lesen versucht. ${ }^{16}$ Ein solches Konzept des Labyrinthischen bezeichnet Sigrid Weigel als „Spurensuche und Textlektüre des Zeichensystems Stadt"17. Die Haltung des Flaneurs ist Ausdruck eines unabhängigen Blicks auf fremde und neuartige Erfahrungswelten, die zum literarischen Stoff werden. ${ }^{18}$ In Episoden tritt der Erzähler bei seinen Annäherungsversuchen an Jerusalem, das im Text metonymisch als Person gedacht (vgl. FJ 137) und als Adressat angesprochen ist (vgl. FJ „Dank“), nur an wenigen Stellen selbst als Figur in Erscheinung. Der literarische Stadtraum steht in Ein Frühling in Jerusalem für ethnische Vielfalt, für drei Weltreligionen und als Ort des Kultur- und Massentourismus. In diesem Labyrinth des Nahen Ostens verkörpert der reisende Erzähler den vereinzelten Menschen in der Masse, der in selbst gewählter Einsamkeit trotz oder gerade wegen der verwirrenden Eindrücke und Einsichten konzentriert und kontemplativ nach Verstehen sucht. 


\section{Zur Metaphorik des Labyrinthischen}

Jerusalem, das seit den Kreuzzügen und den Pilgerfahrten drei Weltregionen als die Himmlische, Heilige Stadt und als Heimstatt gilt, erfährt in Büschers Reisetext eine Würdigung als mythisch stilisierte weibliche Schönheit: „Jerusalem ist eine orientalische Frau. Wer sie sehen will, muß erst durchs Dunkel wandern, lange durch obskure Tunnel irren, durch Gänge und Gewölbe, auf den Abend warten“ (FJ 30). Die Metaphorik des Labyrinthischen setzt den weiblichen Körper als fremden Kontinent dem schwer zu ergründenden und geduldig zu erobernden Stadtkörper Jerusalem gleich. Edward Said folgend erscheint diese bildhafte Formulierung durchaus als eindimensional und orientalisierend. ${ }^{19}$ Trotz dieses Kritikpunktes weist die Textstelle dennoch zurecht auf die Unter- aber auch Abgründigkeit der Metropole Jerusalem als Festungsstadt der Geschichte und Gegenwart hin: „[D]ie Stadt, in ihre Mauer gegürtet (...) drüben am Ölberg Gethsemane, der Garten der letzten Nacht vor der Passion, hinter dem Ölberg die Judäische Wüste. Steinernes Land. Dornen und Fels. Aber der Stein ist fruchtbar. Bilder entspringen, schlägt man nur drauf" (FJ 31). Jerusalem, das topographisch der Dürre abgetrotzt und wegen religiös und kulturell begründeter Alleinvertretungsansprüche gespalten ist, kommt kontrastreich in voller Lebendigkeit (vgl. FJ 46ff.) positiv zur Geltung. Die historische, ethnisch-soziale und politische Komplexität dieser Stadt erscheint dem Erzähler entweder aus der Übersichtsperspektive oder in ihrer Tiefendimension erfassbar zu sein (vgl. FJ 32). Was die Weltmetropole Jerusalem zum historischen und kulturellen Labyrinth und Rätsel macht, sind die historischen und kulturellen Überschreibungen. Dies wird an der Familiengeschichte eines arabischen Hoteliers alten Adels, der um sein Hotel in der Jerusalemer Altstadt kämpfen muss, deutlich gemacht: „'Als Junge hat mein Onkel eine Höhle unter dem Grab König Davids auf dem Zionsberg gekannt. Sie führte tief hinein unter die Stadt, zu immer neuen Höhlen, durch sie konnte man unterirdisch bis unter das Zentrum der Altstadt gelangen’“ (FJ 183). Dieser Gedanke wird in Ein Frühling in Jerusalem an der Grabeskirche (vgl. FJ 117-130) ausgeführt, „eher eine Wirrnis aus Schichten, Zeiten, Räumen, die alle fortlebten“ (FJ 83f.). Analog zu europäischen Städten wie Lemberg, Czernowitz oder Vilnius der k.u.k. Österreich-Ungarn-Region erscheint Jerusalem in 
Büschers Prosaband als transkulturelles Palimpsest der Geschichte ${ }^{20}$ und der Gegenwart. Dies ist möglich, da das Fundament der Stadt aus Zeitschichten von Überschreibungen besteht, die koexistieren und einander gleichzeitig widersprechen können. ${ }^{21}$ In Ein Frühling in Jerusalem bilden diese historischen Schichten einen Resonanzraum, der den Genozid 1915 an den Armeniern durch das Osmanische Reich ${ }^{22}$, das jüdische Exil und die weltweite Diaspora nach 1945 ins Bewusstsein ruft. Was die Weltstadt Jerusalem stark macht, sorgt zugleich für ihre Zerbrechlichkeit und Angreifbarkeit: „Tunnel, die Risse machen, das geht schnell hier, der Untergrund von Jerusalem besteht aus den Ruinen früherer Jerusalems, er ist mit Trümmern verfüllt und porös (...)“ (FJ 175). Hinzu kommt eine komplexe Klanglandschaft ${ }^{23}$ des Sprachengewirrs (vgl. FJ 33f.), eines vielstimmigen Chores (vgl. FJ 41) und „ein[es] vielstimmige[n] Gesang[s]“ (FJ 41) der Konfessionen, die im Zusammenspiel mit dem „Gesang der Wüste, aus den Wadis und felsigen Einöden“ (FJ 41) die Wahrnehmung des reisenden Erzählers in Turbulenz versetzt. Geruchlich bewegen sich seine Eindrücke im „Chaos der Düfte“ (FJ 28) zwischen Gefühlen des Wohlbefindens und des Ekels. Die Palimpsestformation der Stadt Jerusalem zeigt Wirkung sowohl auf kulturelle Orientierungen, „[d]as Arabische ist der Mörtel des Alten Jerusalem“ (FJ 27), als auch auf deren Desorientierung: „Es konnte sein, daß eine Straße als arabische begann, mittendrin jüdisch wurde und als etwas Drittes endete, als äthiopisches Kloster, als syrischer oder maronitischer Konvent" (FJ 138). In Ein Frühling in Jerusalem steht diese Straße metonymisch für die Geschichte der vielfältigen Teilungen Jerusalems zwischen Jordanien wie auch zwischen den arabischen und den jüdischen Israelis seit der Staatsgründung Israels 1948 und darüber hinaus bis 1967 (vgl. FJ 139f).

Eine weitere Deutungsvariante des Labyrinthischen geht in Ein Frühling in Jerusalem auf die Wirkung Jerusalems auf Einheimische und Fremde ein. Während die dort Ansässigen über ein ortsgebundenes Referenzwissen ${ }^{24}$ der Stadttopographie und ihrer architektonischen Besonderheiten verfügen, muss sich der Besucher dieses erst aneignen und „auswendig lernen“25. Auch im jüdischen Viertel der Stadt Jerusalem scheinen ausschließlich Einheimische über die nötigen Ortskenntnisse der Übergänge zu verfügen: „Oder die Tür war nicht auf den ersten Blick zu finden, weil man zu ihr nur durch einen 
gewissen Gang oder Tunnel gelangen konnte, über einen unscheinbaren Treppenaufstieg und so fort" (FJ 138). Die Metaphorik des Labyrinthischen macht den Widerspruch zwischen den Überlebensgeschichten des Holocaust und der nationalistischen Politik Israels gegenüber der palästinensisch-arabischen Bevölkerung nach 1948 sinnbildlich deutlich (vgl. FJ 152f.). Dina, die sich als Aktivistin für eine israelisch-arabische Aussöhnung einsetzte, aber nach den Ereignissen der beiden Intifadabewegungen aufgegeben hat (vgl. FJ 154f.), greift diese ideologisch begründete Problematik auf und schreibt der aggressiven israelischen Siedlungspolitik eine Mitverantwortung an geschehenem Unrecht zu. Der Erzähler nimmt hierzu Stellung: „Ich ahnte, was in ihr kreiste, nein, ich ahnte es nicht, ich sah es vor mir - ein Strudel, der den, der lange hineinschaut, hinabzieht" (FJ 153). Interkulturelle Grenzen konkreter und symbolischer Art verhindern einen gleichberechtigten Umgang zwischen den Ethnien und Kulturen. Hinter Toren (vgl. FJ 47) abgetrennte Stadtviertel repräsentieren territoriale und symbolische Markierungen von "gated communities“26. Solche Räume der Abschottung und Exklusion belegen als historisch zu erklärende Schutzzonen ${ }^{27}$ Grenze nicht als Kontaktzone, vielmehr als sozialen Ausschluss. Dies erläutert die Episode vom arabischen Jungen, der nach einer diskriminierenden Handlung bei einer Passkontrolle überreagiert und einem israelischen Soldaten ein Auge ausschlägt (vgl. FJ 155-158). ${ }^{28}$ Der Erzähler kritisiert, wie militärische und polizeiliche Grenzkontrollen als Ausdruck von Autonomie- und Besitzanspruch, „Landhunger" (FJ 174), gegen soziale Teilhabe anderer Konfessionen und Ethnien (vgl. FJ 185) genutzt werden. Auch nach Sznaider zieht der Diaspora-Nationalismus des Staates Israel zu Unrecht symbolische Grenzen zwischen Ethnien und Kulturen (vgl. FJ 144-148). ${ }^{29}$ Werden Grenzen zur Abschottung gebraucht, ist dies nach Zygmunt Baumann mit einer „Unwilligkeit, sich mit dem Fremden zu mischen, da es gefährlich und riskant“30 erscheint, zu erklären. In Jerusalem werden interkulturelle Konflikte durch Scheingeschäfte (vgl. FJ 174ff) um letzte Grundstücke und Immobilien in der Jerusalemer Altstadt ausgelöst. Samuel P. Huntington bezeichnet diese Art verdeckter Auseinandersetzungen als Bruchlinienkonflikte ${ }^{31}$, die einen Stellvertreterkonflikt zwischen Ethnien und Kulturen schüren. Die Metapher des Labyrinthischen und die episodischen 
Wirklichkeitsbeschreibungen verweisen in Ein Frühling in Jerusalem auf die Komplexität und Vielfalt gesellschaftlichen und kulturellen Zusammenlebens. Interkulturelle und interreligiöse Begegnungen erweisen sich aufgrund von Alleinvertretungsansprüchen auf den Tempelberg mit Felsendom und Grabeskirche (vgl. FJ 203-207, FJ 215) ohne größte beiderseitige Anstrengungen als kaum realisierbar. Dies würde eine Verabschiedung überholter, binärer Denkstrukturen des Kalten Krieges hinsichtlich einer ideologischen Aufteilung nicht nur zwischen Ost und West, sondern auch zwischen dem Westen und dem sogenannten nichtwestlichen ,Rest' der Welt voraussetzen. ${ }^{32}$ Paradoxerweise scheint Jerusalem gerade von dieser Grundspannung aus sowohl behaupteten als auch realen Konfliktlagen zu leben, „[a]ber der große Zünder dort oben funktionierte, das war wieder einmal bewiesen. Er war scharf gestellt, immer“ (FJ 204).

\section{Jerusalem als Projektort ${ }^{33}$ von Transkulturalität}

Ideologische Differenzen (vgl. FJ 93) und soziale Ausgrenzung34 erschweren im heutigen Jerusalem eine Weiterentwicklung von Transkulturalität, obwohl kulturelle Transformationsprozesse infolge von Migrationsbewegungen in der Geschichte dieses Teils des Nahen Ostens vielfach belegt sind:

Erst kamen wenige, dann im vorigen Jahrhundert immer mehr. Und nicht nur aus Europa, nicht nur wegen der Lager, auch aus Marokko, aus dem Irak, von überallher. Gehen Sie freitags zur Klagemauer, die Juden dort beten in ganz verschiedenen Melodien. Sephardisch, orientalisch, amerikanisch. Stellen Sie einen Juden aus Casablanca neben einen aus Lodz, ethnologisch haben Sie nichts gemein. Essen, Kleidung, Sprache sind ganz verschieden. Aber sie lesen die gleiche Thora - das ist es, was sie ausmacht. Darum muß man ihnen, das Orientalische, Russische oder Europäische auch nicht abdressieren. (FJ 220)

Historisch betrachtet sind in dieser Stadt Interreligiosität und Interkulturalität Prinzip, denn „(...) das christliche Herz Jerusalems schlägt in einem orientalischen Körper“ (FJ 28). Im Dazwischen der Kulturen und der Religionen verschwimmen dem Erzähler, „ein[em] Tagträumende[n]“ (FJ 26), die Grenzen zwischen den Kulturräumen und 
Inez Müller

Zeitebenen:

Nur das richtige Türchen mußte ich öffnen und stand im Parlando eines italienischen Klosters, im Weihrauchnebel einer kleinen griechischen Kirche, im Goldglanz eines russischen Nonnenkonvents, im kaiser-und-königlichen Hospiz an der Via Dolorosa oder in einem wahrhaftigen Wiener Kaffeehaus, wo junge Araber warmen Apfelstrudel zur Melange servierten oder eine Tiroler Brotzeit zum Gösser-Bier. (FJ 26f.)

In dieser Bildszene mischen sich Kategorien und kulturelle Zuschreibungen. Ideologisch und kulturell begründete Antagonismen zwischen Ost und West wie der behauptete Gegensatz zwischen einer westlich geprägten und einer nichteuropäischen, anderen ,Rest'welt erscheinen als obsolet und sind im literarischen Text durch eine globalisierte Ess- und Trinkkultur ersetzt. Dennoch bleibt der Erzähler zum Teil eurozentristischer Sichtweise und einem romantisch idealisierenden Stereotyp der OrientOkzident-Konstruktion 35 treu: „Morgenland! So roch es, so klang es, so sah es aus, und im nächsten Moment läuteten Kirchenglocken wie an einem Sonntagmorgen in Köln. Abendland! Auf einmal roch es und klang es und sah sogar aus wie daheim“ (FJ 26). In der Jerusalemer Altstadt sind sowohl Transkulturalität als auch der kulturelle Konflikt gleichzeitig erfahrbar: „Clash der Dünste und Offenbarungen als Normalzustand. Sich kreuzende, ineinander bohrende, einander ignorierende Züge und Prozessionen der gegensätzlichsten Art auf diesem einen Quadratkilometer“ (FJ 28f.). Angesichts dieser widersprüchlichen Wahrnehmungen und Eindrücke erfährt der Erzähler eine prozesshafte Veränderung seiner Wahrnehmungsweisen, was $\mathrm{zu}$ Verschiebungen vorhandener Denkkategorien hinsichtlich Grenze und Kultur ${ }^{36}$ führt:

Ich hatte mich ins Dunkel zwischen den Welten zurückgezogen, ins Grenzgebiet zwischen Rom und Byzanz (...) und meine neue Position hatte den Vorteil, beide zu hören. Im linken Ohr hatte ich den steten Sprechgesang der Lateiner, im rechten Ohr den Sang der Griechen. (...) Ich lag auf der Bank und die Grenze ging mitten durch mich hindurch. (FJ 129)

Die Internalisierung unterschiedlicher kultureller Wahrnehmungen verändert die 
Subjektposition des Ich-Erzählers, der beginnt, das Trennende ${ }^{37}$ zwischen Grenzen, Religionen und Kulturen in Frage zu stellen. In der Shopping Mall Mamilla im Westteil Jerusalems, also an einem Nicht-Ort der Globalisierung meint der Ich-Erzähler, einen Raum der kulturellen Begegnung erkennen zu können, an dem die Grenzen der Herkunft und der Religion bedeutungslos zu sein scheinen (vgl. FJ 52-56). Für die Figur Ada besteht „[d]as Wunder von Mamilla“ (FJ 53; vgl. FJ 51) in einer von ethnischen Konflikten freien multikulturellen Mischung in unbeschwerter Freizeit des Konsums. Ada nimmt mit der metaphorischen Bezeichnung der Einkaufszone als „Berlin-Mitte-Simulation am Jaffator“ (FJ 56) und „Klein-Europa“ (FJ 54) die poetische Setzung eines hybriden, dritten Raumes vor. Die Mamilla Mall ist eine von kommerziellen Interessen geprägte Kontaktzone, die Möglichkeiten des Austausches und der Begegnung bereitstellt.

Wasifs Erzählung vom culture crossing und von sozialer Grenzüberschreitung (vgl. FJ 102f.) während des Jerusalemer huruma-Fests, das in der 1. Hälfte des 20. Jhds. zeitgleich mit dem jüdischen Purimfest in der Osternacht gefeiert wurde, enthält die Utopie einer Rückbesinnung auf die Tradition des friedfertigen Feierns arabischer, jüdischer und christlicher Israelis als karnevalistische Inszenierung der Maskerade und Kostümierung. Das huruma spielte im Rollentausch und gender crossing mit den Möglichkeiten der kulturellen Überschreitung und der Mischung:

'Man verkleidete sich zum Beispiel als Braut und Bräutigam, die Braut trug ein elegantes Kleid, schönes Haar, Make-up und viel Schmuck - aber sie war ein Mann, und umgekehrt. (...) Manche verkleideten sich auch als Juden oder als aschkenasische Juden, sie hatten falsche Locken angelegt. Wieder andere gingen als Bauer und Bauersfrau in der traditionellen Tracht von Ramallah oder in kostbar bestickten Kleidern im Bethlehemer Stil oder als Kosaken mit Pelzhut und Patronenweste oder als Albaner mit rotem marokkanischen Fez [Hervorhebung; I.M.] oder als Diplomat mit Zylinder'. (FJ 102)

Die Wirklichkeit des Jerusalem der Gegenwart erweist sich von Wasifs Erzählversion abweichend als unsicher und belastet. Der fremde Erzähler weigert sich aber, diese historisch verbürgte Utopie einer friedfertigen Koexistenz der Ethnien und Religionen zu 
Inez Müller

verabschieden:

Der schöne Frieden von Jerusalem, den Wasif so warm beschrieb, die gute Nachbarschaft von Juden, Moslems und Christen, die gewiß nicht immer nur harmonisch gewesen war, und doch hatte es sie gegeben - das alles war eine ferne Erinnerung. Er war etwas anderem gewichen, das an manchen Tagen als grauer Staub aus Melancholie auf der Stadt lag, an anderen Tagen knisterte es wie eine glimmende Lunte. (FJ 103)

In Ein Frühling in Jerusalem wird sowohl auf der geschichtlichen Ebene als auch in der Gegenwartsperspektive vom Lebensmodell Diaspora erzählt. Der Begriff der Diaspora schließt im 21. Jhd. die Ausnahmesituation der „gewaltsamen Vertreibung und Zerstreuung über viele verschiedene Zielgebiete“38 des jüdischen Volkes, der armenischen Christen ${ }^{39}$ wie auch von Moslems ${ }^{40}$ ein. Insofern weitet auch Büschers Prosaband den Begriff der Diaspora aus, indem mit der kolonial bedingten Verdrängung der armenischen Bevölkerung aus Westjerusalem in die Altstadt eine historische Kontinuität der Ereignisse von 1915 aufgezeigt ist. Im Erzählerkommentar sind die innerisraelischen Vertreibungsgeschichten als „Schicksalssternschnuppen seither“ (FJ 160) metaphorisch umschrieben. Das Gedenken an die Exil- und Diasporabewegungen nach Jerusalem zeigt Perspektiven auf, an die humanitäre Tradition der Hilfestellung und Rettung (vgl. FJ 76f., 135) anzuschließen, die auch mit diesen Ereignissen verbunden ist. Kennzeichnend für Exilmigranten und diasporische Einwanderer ist, dass deren Gewohnheiten, Identitäten ${ }^{41}$, Geschichten und Erzählweisen ${ }^{42}$ aus ihren Herkunftsländern mit in die Ankunftsländer wandern. In positiver Hinsicht kann die Konstruktion der Diaspora, die für Jerusalem prägend ist und sich aus gemeinsamen Erinnerungen speist, kollektive Identität ${ }^{43}$ stiften und zur Herstellung von politischer Einheit dienen. Dies setzt allerdings voraus, dass kulturelle Identität weder mit Staat und Territorium noch mit nationalistisch konnotierten Symbolen 44 wie Fahnen, Flaggen und Grenzen gleichgesetzt wird:

In der jüdischen Geschichte und Theologie unterscheidet man zwischen Exil (galut) und Diaspora (gola). Während ,Exil' als Strafe aufgefasst wird - ewig wandernd, ewig machtlos -, beinhaltet der Diasporabegriff die Chance, sicher in sich ruhend mit anderen Gemeinschaften zu leben. ${ }^{45}$ 
Dies würde bedeuten, dass sich die Ausrichtung israelischer Politik aus der Identitätsfalle ${ }^{46}$ befreien kann. Das in Ein Frühling in Jerusalem beschriebene globalisierte Geschäftsmodell stellt sich dem Erzähler zumindest im christlichen Viertel Jerusalems als Diasporakonzept zwischen den Ethnien bereits als eine Erfolgsgeschichte dar:

Ein paar Läden, die Antiquitäten, Devotionalien und Alltägliches führten, zwei oder drei Restaurants, die jüdischen Wein von den Golanhöhen und christliches Bier aus dem Westjordanland ausschenkten, der griechische Zahnarzt, der armenische Keramiker, der islamische Friseur (FJ 161).

Der griechische Arzt Dr. Nikos gründet während der 2. Intifada die Jugendfußballmannschaft FC St. Dimitri mit der Auflage, dass in diesem Team Jugendliche christlichen, jüdischen und islamischen Glaubens unter seiner Anleitung trainieren (vgl. FJ 164f.). In Jerusalem macht der Erzähler die Bekanntschaft des Keramikers Elia Kahvedjian (vgl. FJ 165-171), der sich im Gespräch als Nachfahre armenischer Christen mit neuer Identität zu erkennen gibt:

Auch der Keramiker hatte sein kleines Geheimnis, ich kannte es mittlerweile. Die Werkstatt gehörte der einzigen armenischen Familie Jerusalems, die ihren Namen früher einmal arabisiert hatte, was ihr die Familien, die das nicht getan hatten, immer noch nachtrugen. (...) Seine Heimat, die Stadt Urfa im östlichen Anatolien, sah er nie wieder, seine Eltern waren ermordet, seine ganze hundertsechzigköpfige Familie war ausgelöscht. (FJ 165ff.)

In Jerusalem hatte Elias Vater als Überlebender des Völkermords an den armenischen Christen Zuflucht und eine neue Heimat gefunden: „Elia hatte nicht einmal einen Namen gehabt, als er sich hatte nach Jerusalem retten können, nur das nackte Leben. Jerusalem war sein Glück gewesen. Hier war er zum Fotografen des Vorkriegsjerusalems geworden“ (FJ 165f.). Elia war nach dem Verlust der Mutter in kurdischer Obhut „zum Sklaven gemacht" worden (FJ 166; vgl. 166f.). Seiner Wurzeln beraubt legte sich Elia in Jerusalem einen neuen Namen zu: „Es hatte daheim nach Kaffee gerochen. Seine Eltern, sein Vater vielleicht, hatten Kaffee geliebt. Und so nahm er diese kostbare Erinnerung und 
machte sich aus ihr einen Namen, aus dem armenischen Wort für Kaffee, Kahvedjian“ (FJ 167). Diese Erzählung von Identitätsgebung markiert in Ein Frühling in Jerusalem weltgeschichtlich, kulturhistorisch und sprachgeschichtlich relevante Aspekte der Interkulturalität. In den Wirren der 30er- Jahre musste Elia Kahvedjians Familie im Jerusalem unter britischem Mandat eine zweite Verdrängung und Umsiedlung aus Westjerusalem in die Altstadt Jerusalems hinnehmen (vgl. FJ 167). Elia, der Vater des Keramikers Elia Kahvedjian, fertigte als Jerusalemer Fotograf eine Sammlung aus Bilddokumenten ${ }^{47}$ der 20er- und 30er-Jahre an: „Zum Vorschein kam eine ganze Welt - das arabische, christliche, beduinische, jüdische, britisch beherrschte Jerusalem vor dem Krieg“ (FJ 168). Kahvedjians von der Diaspora geprägte Integrationsgeschichte findet in der erzählten Gegenwart des ,neuen' Jerusalem gesetzgeberisch ein Ende, denn Elias Sohn kann wegen der fehlenden Jerusalem ID, „auch blue ID genannt nach ihrer Farbe“ (FJ 169), nicht mit seiner amerikanischen Frau in Jerusalem bleiben. Geltendes Jerusalem-Recht verleiht den Beiden nicht die vollen Staatsbürgerrechte und ist daher Teil einer ethnisch und sozial diskriminierenden wie reglementierenden Einwanderungsgesetzgebung. Die Familiengeschichte der Kahvedjians steht in Ein Frühling in Jerusalem exemplarisch für die widersprüchliche Stadtgeschichte Jerusalems im 20. Jhd., in der sich die Weltgeschichte mit den Ausprägungen der Kolonialzeit, der beiden Weltkriege, der Genozide, der Exodusgeschehnisse und der Staatsgründung Israels widerspiegelt. Der kulturelle Ort Jerusalem zeigt diese Zusammenhänge 1948 und danach verdichtet auf.

\section{Ausblick}

Die Metropole Jerusalem ist nicht losgelöst von den Konfliktlagen um die Siedlungsgebiete im Westjordanland, im Gazastreifen und in der gesamten Region des Mittleren und Nahen Ostens zu sehen. Jerusalem erscheint in einer Ambivalenz zwischen praktizierter Weltoffenheit und der Tendenz zur Grenz- und Selbstabschottung gegen eine als feindlich betrachtete Außenwelt. Diese ambivalente Position zwischen Differenz, Interkulturalität aber auch der Transkulturalität macht für den reisenden Erzähler in Ein Frühling in Jerusalem den Reiz dieses Stadtraumes in permanenter Veränderung und in 
latenter Konfliktlage aus. Dabei kann die Erinnerung an positive Folgewirkungen der Diaspora nach 1945 und den forcierten Migrationsbewegungen seit den 90er-Jahren durch Globalisierungsprozesse in einer Gesellschaftsformation zwischen den Kulturen durchaus identitätsstiftend und innovativ wirken, wovon der Erzählband berichtet. Aus Büschers Erzählungen spricht eine verhalten optimistische Sicht auf das kulturelle Zusammenleben in Jerusalem. Homi K. Bhabha formuliert die positiven Chancen von Globalisierungstransformationen im 21. Jhd. in noch pointierterer Weise:

In mir ist die Hoffnung, dass nach den Wirren von Kriegen, Besatzungen, Segregationen und Vertreibungen diese ungerichteten Energien gefundener und verlorener Orte sich zu einem Konzept für ein Leben mit gemeinsamen Grenzen und kontrapunktischen Geschichten zusammenfinden. Wenn Unterdrückung und Zerstörung Mauern niederreißen und Grenzen auflösen können, weshalb sollten in Zeiten des Friedens die Tore nicht offen bleiben können, die Räume neu besiedelt werden? ${ }^{48}$

Der Erzähler in Ein Frühling in Jerusalem würde diesen Optimismus gerne teilen können, stattdessen gibt ihm die Wirklichkeit im Nahen Osten Anlass zu entgegengesetzten Schlussfolgerungen, denn culture clashes und Besitzansprüche neokolonialistischer Ausprägung belasten Prozesse einer Annäherung:

Man konnte den Haredim, den Ultraorthodoxen, vieles vorwerfen, aber nicht fehlenden Sinn für die Heiligkeit der Heiligen Stadt, das ganz gewiß nicht. Jetzt hätte ich gern [sic] Ada am Tisch gehabt, die genau das, wovor ihm graute, ein Wunder genannt hatte - die Europäisierung, einen europäischen Ort wie die Mamilla Mall, eine sacht entheiligte Heilige Stadt. (FJ 179)

Am erzählten Ort Jerusalem dominiert ethnische, gesellschaftliche, religiöse und ideologische Spaltung und somit die Wahrnehmung von Differenz. Ob das Modell ${ }^{49}$ einer pluralistisch-demokratisch ausgerichteten Zivilgesellschaft für Jerusalem und Israel geeigneter wäre, bleibt im Prosaband Ein Frühling in Jerusalem offen. 


\section{Bibliografie}

Bashir, Bashir (2012), „Politik der Versöhnung“, in: Lebensmodell Diaspora. Über moderne Nomaden. Isolde Charim / Gertraud Auer Borea (eds.) (2012). Bielefeld, Transcript, 227235.

Bauböck, Rainer (2012), „Diaspora und transnationale Demokratie“, in: Lebensmodell Diaspora. Über moderne Nomaden. Isolde Charim / Gertraud Auer Borea (eds.) (2012). Bielefeld, Transcript, 19-33.

Baumann, Zygmunt (2012), „Leben in der Diaspora“, in: Lebensmodell Diaspora. Über moderne Nomaden. Isolde Charim / Gertraud Auer Borea (eds.) (2012). Bielefeld, Transcript, 95-103.

Benjamin, Walter (1983³), Das Passagen-Werk. Erster Band. Rolf Tiedemann (ed.) (1983³). Frankf./M, Suhrkamp.

Bhabha, Homi K. (2012), „Globalisierung und Ambivalenz“, in: Lebensmodell Diaspora. Über moderne Nomaden. Isolde Charim / Gertraud Auer Borea (eds.) (2012), 54-64.

Transkulturalität - Türkisch-deutsche Konstellationen in Literatur und Film. Hendrik Blumentrath / Julia Bodenburg / Roger Hillmann / Martina Wagner-Egelhaaf (eds.) (2007). Münster, Aschendorff.

Wolfgang Büscher (2014²), Ein Frühling in Jerusalem. Berlin, rowohlt Berlin.

Lebensmodell Diaspora. Über moderne Nomaden. Isolde Charim / Gertraud Auer Borea (eds.) (2012). Bielefeld, Transcript.

Ebeling, Knut (2010), „Historischer Raum: Archiv und Erinnerungsort“ in: Stephan Günzel unter Mitarbeit von Franziska Kümmerling (ed.) (2010), Raum. Ein interdisziplinäres Handbuch. Stuttgart / Weimar, Metzler, 121-133.

Ette, Ottmar (2011), „Urbanität und Literatur. Städte als transareale Bewegungsräume bei Assia Djebar, Emine Sevgi Özdamar und Cecile Wajsbrot“, in: Markus Messling / Dieter Läpple / Jürgen Trabant (eds.) (2011), Stadt und Urbanität - Transdisziplinäre Perspektiven. Berlin, Kadmos, 221-246.

Goebel, Rolf J. (2001), Benjamin Heute. Großstadtdiskurs, Postkolonialität und Flanerie 
zwischen den Kulturen. München, Iudicium.

Hamann, Christof (2001), Grenzen der Metropole - New York in der deutschsprachigen Gegenwartsliteratur. Wiesbaden, Deutscher Univ.-Verlag.

Hintermeier, Hannes (2014), „Ich bin hier, weil es Jerusalem ist“, F.A.Z. v. 29.11.2014. Manuskript 4 Seiten.

Huntington, Samuel P. (19976), Der Kampf der Kulturen - Die Neugestaltung der Weltpolitik im 21. Jahrhundert. München / Wien, Europa-Verl.

Illouz, Eva (2015), „Lasst sie nicht gewinnen! Wer wissen will, wie mit einem feindlichen Umfeld zu verfahren ist, schaue nach Israel“, Die Zeit v. 19.11. 2015.

Jerusalem through my father's eyes. Illustrated book. Kevork Kahvedjian (ed.) (2005) o.0., Elia Photo Service.

Judt, Tony (2012), „Ist Israel (noch) gut für die Juden?“, in: Charim / Auer Borea (eds.) (2012), 207-214.

Kulturen in Bewegung. Beiträge zur Theorie und Praxis der Transkulturalität. Dorothee Kimmich / Schamma Shahadat (eds.) (2012). Bielefeld, Transcript.

Klingst, Martin (2015), „An den Grenzen der Humanität. Befestigungen verschaffen eine Atempause und sorgen für neue Probleme“. Die Zeit v. 29.10.2015.

Loewy, Hanno (2012), „Warum Israel die Diaspora neu begründet“, in: Charim / Auer Borea (eds.) (2012), 195-205.

Makarska, Renata (2012), „Kakanien der neuen Generation. Zentraleuropa zwischen Transkulturalität und Differenz", in: Kulturen in Bewegung. Beiträge zur Theorie und Praxis der Transkulturalität. Dorothee Kimmich / Schamma Shahadat (eds.) (2012). Bielefeld, Transcript, 235-260.

Stadt und Urbanität - Transdisziplinäre Perspektiven. Markus Messling / Dieter Läpple / Jürgen Trabant (eds.) (2011). Berlin, Kadmos.

Özturk, Kadriye (2007), „Ida Hahns Orientalische Briefe als konfliktreiche Grüße”, in: JeanMarie Valentin (ed.) (2007), Divergente Kulturräume in der Literatur - Kulturkonflikte in der Reiseliteratur. Internationaler Germanistenkongress 11, Paris. Bern, Lang, 351-357.

Said, Edward (20144), Orientalismus. Frankf./M., Fischer. 
Sen, Amartya (20073), Die Identitätsfalle. Warum es keinen Krieg der Kulturen gibt. München, Beck.

Sznaider, Natan (2012), „Diaspora-Nationalismus - Jüdische Erfahrungen und universale Lehren“, in: Charim / Auer Borea (eds.) (2012), Bielefeld, 186-193.

Chakravorty Spivak, Gayatri (2012), „Außerhalb in der Metropole?“, in: Charim / Auer Borea (eds.) (2012), 65-73.

Trojanow, Ilija (2012), „Exil als Heimat - Die literarischen Früchte der Entwurzelung“, in: Charim / Auer Borea (eds.) (2012), 155-163.

Weigel, Sigrid (2011), „Text und Topographie der Stadt - Symbole, religiöse Rituale und Kulturtechniken in der europäischen Stadtgeschichte", in: Stadt und Urbanität Transdisziplinäre Perspektiven. Markus Messling / Dieter Läpple / Jürgen Trabant (eds.) (2011). Berlin, Kadmos, 185-220.

Wolfgang Welsch (2012), „Was ist eigentlich Transkulturalität?“. In: Kulturen in Bewegung. Beiträge zur Theorie und Praxis der Transkulturalität. Dorothee Kimmich / Schamma Shahadat (eds.) (2012). Bielefeld, Transcript, 25-40.

PD Dr. Inez Müller, seit 2010 an der Universität Paderborn, Institut für Germanistik und Vergleichende Literaturwissenschaft, Lehrstuhl Prof. Dr. Michael Hofmann, 1993 Promotion zu Brecht und Benjamin an der Universität des Saarlandes, 1993-2008 lecturer und senior lecturer an den schwedischen Universitäten Luleå, Halmstad, Umeå und Växjö, 2006 schwedische Habilitation (associate professor), seit 1999 Mitarbeit an der Schriftenreihe des Instituts für deutsche Gegenwartsliteratur und Deutschlandstudien (DGD) der Universität Göteborg: „Zur Darstellung von Zeitgeschichte in deutschsprachiger Gegenwartsliteratur" (I-IX). 


\section{Fussnoten}

${ }^{1}$ Wolfgang Büscher: Ein Frühling in Jerusalem. Berlin $2014^{2}$ (Im Fließtext als Sigle FJ mit Seitenzahl geführt).

2 Entgegen Hannes Hintermeiers Zuschreibung als „erzählendes Sachbuch“ wird im vorliegenden Beitrag Ein Frühling in Jerusalem als literarischer Text zwischen den Genres Reiseliteratur, Großstadterzählung und autobiographischem Schreiben betrachtet. Vgl. Hannes Hintermeier: „Ich bin hier, weil es Jerusalem ist“. In: F.A.Z. v. 29.11.2014. Manuskript 4 Seiten. Hier: S. 2.

3 Vgl. Christof Hamann: Grenzen der Metropole - New York in der deutschsprachigen Gegenwartsliteratur. Wiesbaden 2001. Hier: S. 12-32.

${ }^{4}$ Ottmar Ette: „Urbanität und Literatur. Städte als transareale Bewegungsräume bei Assia Djebar, Emine Sevgi Özdamar und Cecile Wajsbrot“. In: Stadt und Urbanität - Transdisziplinäre Perspektiven. Hg. v. Markus Messling / Dieter Läpple / Jürgen Trabant. Berlin 2011, S. 221-246. Hier: 213.

5 Ibidem, S. 26.

${ }^{6}$ Vgl. ibidem, S. 21.

${ }^{7}$ Vgl. Wolfgang Welsch: „Was ist eigentlich Transkulturalität?“. In: Kulturen in Bewegung. Beiträge zur Theorie und Praxis der Transkulturalität. Hg. v. Dorothee Kimmich / Schamma Shahadat. Bielefeld 2012, S. 25 -40.

8 Kadriye Özturk: „Ida Hahns Orientalische Briefe als konfliktreiche Grüße”. In: Divergente Kulturräume in der Literatur - Kulturkonflikte in der Reiseliteratur. In: Internationaler Germanistenkongress 11, Paris. Hg. JeanMarie Valentin. Bern 2007, S. 351-357. Hier: S. 355.

9 Vgl. Transkulturalität - Türkisch-deutsche Konstellationen in Literatur und Film. Hg. v. Hendrik Blumentrath / Julia Bodenburg / Roger Hillmann / Martina Wagner-Egelhaaf. Münster 2007.

10 Natan Sznaider: „Diaspora-Nationalismus - Jüdische Erfahrungen und universale Lehren“. In: Lebensmodell Diaspora. Über moderne Nomaden. Hg. v. Isolde Charim / Gertraud Auer Borea. Bielefeld 2012, S. 186-193. Hier: $191 \mathrm{ff}$.

11 Die Soziologin Illouz erklärt diese Tendenz mit einer „Sicherheitsfixierung“ des israelischen Staats. Vgl. Eva Illouz: „Lasst sie nicht gewinnen! Wer wissen will, wie mit einem feindlichen Umfeld zu verfahren ist, schaue nach Israel“. In: Die Zeit v. 19.Nov. 2015.

12 Vgl. Kimmich / Shahadat (2012), „Einleitung“, S. 7-21. Hier: S. 13.

13 Vgl. Özturk (2007), S. 354.

14 Vgl. Blumentrath / Bodenburg / Hillman / Wagner-Egelhaaf (2007), S. 118f.; Hanno Loewy: „Warum Israel die Diaspora neu begründet“. In: Charim / Auer Borea (2012), S. 195-205. Hier: S. $196 f$. 
15 Walter Benjamin: Das Passagen-Werk. Erster Band: M [Der Flaneur]. Hg. v. Rolf Tiedemann. Frankf./M. 1983. 3. Auflage, S. 524-569.

16 Vgl. Rolf J. Goebel: Benjamin Heute. Großstadtdiskurs, Postkolonialität und Flanerie zwischen den Kulturen. München 2001, S. 134ff.

17 Sigrid Weigel: „Text und Topographie der Stadt - Symbole, religiöse Rituale und Kulturtechniken in der europäischen Stadtgeschichte. In: Stadt und Urbanität - Transdisziplinäre Perspektiven. Hg. v. Markus Messling / Dieter Läpple / Jürgen Trabant. Berlin 2011, S. 185-220. Hier: S. 190.

18 Vgl. Benjamin (1983), S. 554, S. 525.

19 Auch an anderer Stelle erweist sich der Erzähler als nicht frei von stereotyper kultureller Zuschreibung: „Wenn es hart auf hart kam, wenn es um Blut und Geld ging, dann dachte Charly Effendi als Mann des Orients“ (FJ 158). Kulturelle Werturteile negativer Art sind aus einer Basarszene herauszulesen: „Einmal hineingelockt in einen dieser schmalen, aber oft tiefen Läden, fällt es dem Nichtorientalen in seiner skrupulösen Unbeholfenheit schwer, freizukommen, die Händler wissen das - die jahrtausendealte Basarschläue der Heiligen Stadt" (FJ 19).

20 Vgl. Renata Makarska: „Kakanien der neuen Generation. Zentraleuropa zwischen Transkulturalität und Differenz". In: Kulturen in Bewegung. Beiträge zur Theorie und Praxis der Transkulturalität. Dorothee Kimmich / Schamma Shahadat (eds.) (2012). Bielefeld, S. 235-260. Hier: S. 244f.

21 Vgl. Özturk (2007), S. 355.

22 Vgl. Samuel P. Huntington: Der Kampf der Kulturen - Die Neugestaltung der Weltpolitik im 21. Jahrhundert. München / Wien 19976, S. 460.

${ }^{23}$ Ette (2011), S. 223.

24 Vgl. zu Benjamins Poetologie des topographical turn: Knut Ebeling: „Historischer Raum: Archiv und Erinnerungsort". In: Raum. Ein interdisziplinäres Handbuch. Hg. v. Stephan Günzel unter Mitarbeit von Franziska Kümmerling. Stuttgart / Weimar 2010, S. 121-133. Hier: S. 122-126.

25 Weigel (2011), S. 190.

26 Zygmunt Baumann (2012): „Leben in der Diaspora“. In: Charim / Auer Borea, S. 95-103. Hier: S. 102.

27 Der Staat Israel hat seit seiner Gründung keine topographisch definierte Außengrenze. Vgl. Tony Judt (2012): „Ist Israel (noch) gut für die Juden?“. In: Charim / Auer Borea, S. 207-214. Hier: S. 208. 
${ }^{28}$ Publizistisches zu Israels Zäunen und Mauern im Westjordanland, Gazastreifen und zu Ägypten: vgl. Martin Klingst: „An den Grenzen der Humanität. Befestigungen verschaffen eine Atempause und sorgen für neue Probleme“. In: Die Zeit v. 29.10.2015.

${ }^{29}$ Vgl. Sznaider (2012), S. 190ff.

30 Baumann (2012), S. 102.

31 Huntington (19976), S. 445.

32 Vgl. idem, S. 20.

33 Entgegen der hier vorgenommenen Kennzeichnung als Transkulturalität kennzeichnet Tony Judt den Staat Israel in erster Linie als Projektort der Rückholung der weltweit zerstreuten Juden: Vgl. idem (2012), S. 208.

34 Bashir verweist auf die verschärfte Problematik Israels, die sich aus sozialer Ausgrenzung und der Unterordnung der palästinensischen Israelis durch die israelische Militärverwaltung bis 1966 historisch ergeben hat: Bashir Bashir (2012): „Polititik der Versöhnung“. In: Lebensmodell Diaspora. Über moderne Nomaden. Isolde Charim / Gertraud Auer Borea (eds.) (2012). Bielefeld, S. 227-235. Hier: S. 229.

35 Vgl. Edward Said: Orientalismus. Frankf./M. 20144.

${ }^{36}$ Vgl. Hamann (2001), S. 247, auch S. 188.

37 Vgl. idem, S. 188.

38 Rainer Bauböck (2012): „Diaspora und transnationale Demokratie“. In: Charim / Auer Borea. Bielefeld 2012, S. 19-33. Hier: S. 19; vgl., S. 21.

${ }^{39}$ Am 17.12.15 wurde von Prof. Dr. Michael Hofmann an der Universität Paderborn, Institut der Germanistik und Vergleichenden Literaturwissenschaft die Armin T. Wegner - Forschungsstelle eröffnet. Im Juni 2015 fand vorab eine Tagung zum Thema „Türken und Armenier 1915 - „Aghet“: Totenklage und Gedächtnis des Schreckens" statt.

40 Vgl. Gayatri Chakravorty Spivak (2012): „Außerhalb in der Metropole?“. In: Charim / Auer Borea, S. S. 6573. Hier: S. 68.

41 Vgl. Baumann (2012), S. 99.

42 Vgl. Ilija Trojanow (2012): „Exil als Heimat - Die literarischen Früchte der Entwurzelung“. In: Isolde Charim / Gertraud Auer Borea, S. 155-163. Hier: S. 157.

${ }^{43}$ Vgl. Bauböck (2012), S. 19f.; Loewy (2012), S. 202.

${ }^{44}$ Vgl. Huntington (19976), S. 18.

N.o 34 - 06/ 2016 | 91-110 - ISSN 1645-1112 | http:/dx.doi.org/10.21747/16451112/litcomp34a7 
Inez Müller

45 Sznaider (2012), S. 186; vgl. S. 187.

${ }^{46}$ Vgl. Amartya Sen: Die Identitätsfalle. Warum es keinen Krieg der Kulturen gibt. München $2007^{3}$, S. 9-12, auch S. $26 \mathrm{ff}$.

47 Jerusalem through my father's eyes. Illustrated book. Ed. by Kevork Kahvedjian, o.0. 2005.

${ }^{48}$ Homi K. Bhabha (2012): „Globalisierung und Ambivalenz“. In: Charim / Auer Borea, S. 54-64. Hier: S. 64.

49 Vgl. Bashir (2012), S. 229, S. 234f. 